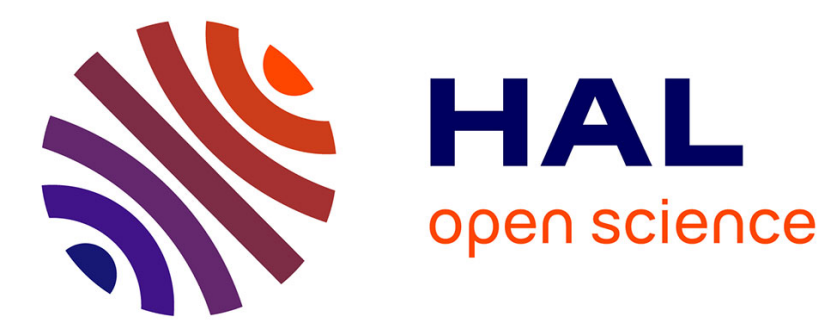

\title{
Essai d'interprétation des propriétés magnétiques du sesquioxyde de fer rhomboédrique
}

Louis Néel

\section{To cite this version:}

Louis Néel. Essai d'interprétation des propriétés magnétiques du sesquioxyde de fer rhomboédrique. Annales De Physique, 1949, 12 (4), pp.249-268. 10.1051/anphys/194912040249 . hal-02888374

\section{HAL Id: hal-02888374 \\ https://hal.science/hal-02888374}

Submitted on 29 Jul 2020

HAL is a multi-disciplinary open access archive for the deposit and dissemination of scientific research documents, whether they are published or not. The documents may come from teaching and research institutions in France or abroad, or from public or private research centers.
L'archive ouverte pluridisciplinaire HAL, est destinée au dépôt et à la diffusion de documents scientifiques de niveau recherche, publiés ou non, émanant des établissements d'enseignement et de recherche français ou étrangers, des laboratoires publics ou privés. 


\title{
ESSAI D'INTERPRÉTATION DES PROPRIÉTÉS MAGNÉTIQUES DU SESQUIOXYDE DE FER RHOMBOÉDRIQUE
}

\author{
Par M. Louis NÉEL
}

Professeur à la Faculté des Sciences de Grenoble.

SOMMAIRE. - Après avoir rappelé les propriétés magnétiques essentielles du sesquioxyde de fer rhomboédrique, l'auteur montre qu'elles peuvent s'ınterpréter par la superposition d'un antiferromaqnétisme et d'un faible ferromagnétisme. L'antiferromagnétisme est une propriété spécifique du sesquioxyde pur et ressemble dans ses manifestations à celui des autres antiferromagnétiques connus, $\mathrm{MnO}, \mathrm{EeO}$, etc...; il est notamment responsable des anomalies de chaleur spécifique et de dilatation. Le ferromagnétisme est dù à une impureté, dont la proportion est del'ordre du centième.

Cette impureté est probablement de la magnétite qui, soluble à haute température dans le sesquioxyde, se précipite au cours du refroidissement, apres formation d'une phase intermédiaire non cubique, orientée par rapport au réseau cristallin du sesquioxyde, en accord direct avec l'existence des groupements épitaxiques spinelle-oligiste étudiés en minéralogie et selon un mécanisme analogue à celui qui donne naissance aux structures de Widmanstátten et en particulier au durcissement structural du système $\mathrm{Al}-\mathrm{Ag}$. On rend compte ainsi de l'orientation des propriétés ferromagnétiques par rapport au réseau cristallin du sesquioxyde. La coīncidence du point de Curie ferromagnétique avec le point de transition antiferromagnétique à ${ }_{67} 7^{\circ} \mathrm{C}$ provient des couplages magnétiques de Weiss-Heisenberg liés à la continuité du réseau cristallin de la phase intermédiaire et du réseau cristallin du sesquioxyde.

Enfin certaines propriétés, comme la grandeur du champ coercitif, l'aimantation à chaud, etc., proviennent simplement de la grande finesse des particules ferromagnétiques.

1. Les deux variétés de sesquioxy le de fer $\left(^{1}\right)$. - L'oxyde ferrique, $\mathrm{Fe}_{2} \mathrm{O}_{3}$, ou sesquioxyde de fer, est un corps dimorphe qui cristallise soit dans le système rhomboédrique $\left(\mathrm{Fe}_{2} \mathrm{O}_{3} \alpha\right)$, soit dans le système

(1) Cf. par ex. l'article de G. Chaudron dans le Traité de Chimie Minérale de Pascal, 9, 728 .

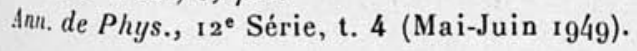


cubique $\left(\mathrm{Fe}_{2} \mathrm{O}_{3} \gamma\right)$. La forme cubique est instable et se transforme, entre $400^{\circ} \mathrm{C}$ et $600^{\circ} \mathrm{C}$, en $\mathrm{Fe}_{2} \mathrm{O}_{3} \alpha$ La forme rhomboédrique est extrêmement répandue à l'état naturel, notamment en cristaux d'hématite (ou fer oligiste) de grandes dimensions. C'est un con-tituant important des roches, notamment des laves, des argiles et des pro. duits dérivés : poteries, briques, etc... Les propriétés magnétiques de tous ces corps sont fort singulières. Quoique dépourvues d'applications industrielles, elles présentent cependant une grande importance tant par des difficultés d'interprétation qui n'ont pas encore été résolues qua par l'application qui en a été faite pour rechercher les variations dans le passé de la direction et de l'intensité du champ magnétique terrestre.

2. Les propriétés magnétiques du sesquioxyde de fer. - Les deux variétés sont ferromagnétiques : les propriétés de la variété cubique sont normales et se rapprochent beaucoup de celles de la magnétite; c'est ainsi que sou aimantation spécitique à saturation, au zéro absolu, est égale, d'après P. Weiss et R. Forrer (', à 83,5 u. é. m., celle de la magnétite étant de $9^{8,2} \mathrm{u}$. é. $\mathrm{m}$.

De soo côté, le sesquioxyde rhomboédrıque présente également les caractéristiques essentielles du ferromagnétisme : hystérèse, rémanence, point de Curie situé à $67^{\circ} \mathrm{C}$. Le champ coercitif peut atteindre des valeurs fort élevées, jusqu'à 7600 œrsteds $\left({ }^{2}\right)$. Dans les champs faibles et moyens vis- $\mathrm{a}$-vis du champ coercitif apparaissent des propriétés magnétiques remarquables que nous étudierons ultérieurement et qui, en dernière analyse, paraissent provenir simplement d'un état d'extrême division de la matière ferromagnétique. Cependant, le caractère magnétique le plus frappant de ce corps est la faible valeur de son aimantation spécifique à saturation $\sigma_{\dot{s}}$ qui est de l'ordre de o, 5 u. é. m., c'est-à-dire cent fois plus petite que celle des ferromagnétiques normaux $\left({ }^{3}\right)$; en outre elle varie d'un échantillon à l'autre.

3. L'hypothèse de l'impureté ferromagnétique. - A première vue, il paraît donc assez tentant d'atıribuer le magnétisme de $\mathrm{Fe}_{2} \mathrm{O}_{3}$ xà la présence d'une impureté ferromagnétique : par exemple à des traces de $\mathrm{Fe}_{2} \mathrm{O}_{3} \gamma$ non transformé dont le point de Curie est précisément voisin de $675^{\circ} \mathrm{G}\left({ }^{4}\right)$. Cette hypothèse est confirmée par les travaux de

(1) Ann. de Phys., 1929, 12, 279.

$\left(^{2}\right)$ J. Ropuet. C. R. Ac. Sc., 1947, 224, 1418.

(3) T. Townsend Smith. Phys. Rev., 1916, 8, 721; R. Chevalliea el S. Mathieu. Ann. de Phys., I943, 18, 258.

(4) A. Michel. Ann. de Chimie, $1937,8,317$. 
J. L. Snoëk ( $\left.{ }^{1}\right)$ qui, en chauffant d'une manière prolongée $\mathrm{Fe}_{2} \mathrm{O}_{3} \alpha$ dans l'oxygène à $\mathrm{I} 200^{\circ} \mathrm{C}$, a réussi à diminuer son aimantation spécifique maximum jusquà 0,20 u. é. $m$., en partant d'une valeur initiale de 0,93 .

Cependant cette explication ne rend comple que d'une petite partie des propriétés de $\mathrm{Fe}_{2} \mathrm{O}_{3} \alpha$. D'autres propriétés sont certainement spécifiques de la substance pure et ne peuvent, à cause de leur grande intensité, être raisonnablement attribuées à des impuretés. C'est ainsi que G. Chaudron et $\mathrm{H}$. Forestier $\left({ }^{2}\right)$ ont montré que $\mathrm{Fe}_{2} \mathrm{O}_{3} \alpha$ possédait des anomalies dilatométriques, avec discontinuité du coefficient de dilatation à $67^{\circ} 5^{\circ} \mathrm{C}$, qui présentent la même allure et la même amplitude que celles de la magnétite. De la même fuçon, la chaleur spécifique, mesurée par Furnas $\left({ }^{3}\right)$ et par Roth et Bertram $\left({ }^{4}\right)$, comparée à la chaleur spécifique de corps analorues, montre que $\mathrm{Fe}_{2} \mathrm{O}_{3} \alpha$ pussede une anomalie de chaleur spécifique d'un ordre de grandeur comparable à celle des ferromagnétiques ordinaires. Enfin, l'ćtude des propriétés magnétiques au-dessus du point de Curie révèle l'existence d'un paramagnétisme important qui, selon A. Serres $\left({ }^{5}\right)$, est indépendant de la température entre $700^{\circ} \mathrm{C}$ et $750^{\circ} \mathrm{C}$ et correspond à une susceptibilité spécifique $\chi$ de $19,7 \cdot 10^{-6}$. D'après des mesures plus récentes de $\mathrm{K}$. Endô $\left({ }^{6}\right)$, poussées jusqu'à $1100^{\circ} \mathrm{C}$, il s'agit plutôt d'un paramagnétisme variable avec la température selon la loi de Weiss :

$$
\chi=\frac{0,085}{1+4000}
$$

où $t$ est la température centigrade. La constante de Curie atomique correspondante est d'environ 6,8 . Eutin, il ressort de l'étude approfondie que R. Chevallier et S. Mathieu ( ${ }^{7}$, ont consacrée aux pro. priétés magnétiques des poudres d'béınatite à la température ordinaire que tout se passe comme si un faible ferromagnétisme, variable d'un échantillon à l'autre, se trouvait superposé à un paramagnétisme de susceptibilité $\chi=20 \cdot 10^{-6}$.

Le rapprochement de ces différents faits conduit donc à attribuer les propriétés magnétiques du sesquioxyde rhomboédrique ordinaire à la superposition d'un antiferromagnétisme qui serait celui du

() Physica, 1936, 3, 463.

(') C. R. Ac. Sc., 1924. 179, 763 .

(3) Trans. Amer. Inst. Chem. Ing., 1926, 18, 3o9; voir aussi : Krause. $Z$. Anorg. Chem., 1929, 180, 120.

(4) Z. Elelctrochemie, $19^{2} 9,35,306$.

(5) Ann. de Phys., 1932, 17, 5.

(6) Sc. Rep. Tóhoku Imp. Univ., ${ }^{1} 9^{3} 7,25,879$.

(') Ann. de Phys., 1943, 18, 258. 
sesquioxyde pur et d'un faible ferromagnétisme dû à une impureté, par exemple à un état du fer moins oxygéné que $\mathrm{Fe}_{2} \mathrm{O}_{3}$.

\section{Propriétés magnétiques provenant de l'antiferromagnétisme} fondamental de $\mathrm{Fe}_{2} \mathrm{O}_{3} \alpha$ pur. - Ce sont :

a) à haute température, le paramagnétisme variable avec la température exprimé par la relation $[\mathbf{I}]$;

b) à basse température, le paramagnétisme sous-jacent, de susceptibilité égale à $20 \cdot 10^{-6}$, mis en évidence par Chevallier et Mathieu ;

c) l'anomalie de dilatation et l'anomalie de chaleur spécifique, avec une discontinuité à $67^{\circ} \mathrm{C}$.

Effectivement, on recounaît là les propriètés caractéristiques essentielles de l'antiferromagnétisme, telles qu'elles ont été prévues par les théories de L. Néel $\left({ }^{1}\right)$, F. Bitter $\left({ }^{2}\right)$ et J. H. Van Vleck $\left({ }^{3}\right)$ et observées par Bizette et Tsaï ( $\left.{ }^{4}\right)$ sur $\mathrm{MnO}, \mathrm{FeO}, \mathrm{MnF}_{2}, \mathrm{FeF}_{2}$, etc... Le porteur de magnétisme est ici l'ion ferrique $\mathrm{Fe}^{+++}$. Très vraisemhlablement, selon une ancienne idée due à R. Forrer, ces ions sont rangés par couches successives perpendiculaires à l'axe ternaire, aimantées spontanément et alternativement suivant les sens opposés $\left({ }^{5}\right)$ d'une même direction qui est la direction d'antiferromagnétisme. La constante de Curie atomique, au-dessus du point de transition de $675^{\circ} \mathrm{C}$, devrait ainsi avoir la valeur de 4,4 que la théorie assigne aux sels ferriques : or, l'expérience donne 6,8 . Il ne faut pas cependant attacher trop d'importance à cette discordance : d'une part, cette valeur provient de mesures faites relativement près du point de transition et il faut craindre, comme dans le cas des ferromagnétiques, les anomalies dues aux fluctuations du champ moléculaire; les expériences de A. Serres $\left({ }^{6}\right)$ sur l'antiferromagnétique MnTe mettent d'ailleurs bien en évidence cette augmentation apparente de la constante de Curie au voisinage du point de transition. D'autre part, conme le point de transition antiferromagnétique est très élevé, il y a à craindre de fortes corrections de dilatation, provenant de la variation thermique du champ moléculaire, selon le mécanisme étudié autrefois par L. Néel ( $\left.{ }^{7}\right)$.

(1) Ann. de Phys., 1932, 17, 5: J. de Phys., 1932, 3, 160; Ann. de Phys., 1936, 5, .232; C.R. Ac. Sc., 1936, 203, 304.

$\left.{ }^{2}\right)$ Phys. Reo., 1938, 54, 79 .

(3) J. chem. Phys., 1941, 9, 85.

(4) Ann. de Phys., 1946, 1, 223.

(5) L. NÉel. Ann. de Phys., 1948, 3, 137 .

(6) J. de Phys., $1947,8,146$.

(7) Ann. de Phys., $19^{37}, 8,237$; C. R. Ac. Sc., 1936, 202, 1028. 
5. Propriétés magnétiques provenant de l'impureté ferromagnétique. -Il s'agit du faible ferromagnétisme signalé dans le paragraphe 2. La faible saturation spécifique qui est de l'ordre de $0,5 \mathrm{u}$. é. $\mathrm{m}$. montre que la proportion de l'impureté doit être de l'ordre du centième. L'hystérèse accentuée ( $\left.{ }^{1}\right)$ montre que l'impureté est dans un état d'extrême division, ou bien qu'elle est soumise à des contraintes mécaniques intenses. D'autre part les propriétés dans les champs très faibles, que nous étudierons dans un Mémoire ultérieur $\left({ }^{2}\right)$, indiquent également un état de grande division qui, somme toute, paraît assez normal pour une impureté dont la proportion relative dans l'échantillon est faible.

Cependant, certaines expériences montrent l'existence d'étroites relations entre l'impureté et le sesquioxyde lui-même, relations dont la simple hypothèse de l'impureté ne rend aucunement compte.

Il y a d'abord ce fait remarquable que le point de disparition des propriétés ferromagnétiques de l'impureté présumée coïncide à ${ }_{67} 5^{\circ} \mathrm{C}$ avec le point de transition antiferromagnétique de $\mathrm{Fe}_{2} \mathrm{O}_{3} \alpha$ pur : serait-ce simplement un hasard?

Il s'agit aussi et surtout d'anciennes expériences de Townsend Smith $\left(^{3}\right)$ sur l'aimantation d'un monocristal d'hématite suivant les différentes directions. Suivant l'axe ternaire, les cristaux paraissent simplement paramagnétiques, avec une susceptibilité spécifique de $21.10^{-6}$. Au contraire, dans le plan perpendiculaire à l'axe ternaire, ils sont ferromagnétiques et la forme de la courbe d'aimantation suggère la superposition d'un paramagnétisme du même ordre de grandeur que le précédent et d'un ferromagnétisme correspondant à une aimantation à saturation spécifique variable de 0,2 à $0,4 \mathrm{u}$. é. m., selon les échantillons. En somme, tout se passe comme si l'impureté possédait une direction de très difficile aimantation coïncidant avec l'axe ternaire du cristal d'hématite dans lequel elle est incluse; l'impureté est donc orientée par son support.

6. L'impureté considérée comme un précipité de magnétite orienté par la matrice. - Il semble résulter des expériences de Snoek (cf. § 3) que le ferromagnétisme de $\mathrm{Fe}_{2} \mathrm{O}_{3} \alpha$ soit produit par un déficit d'atomes d'oxygène par rapport à la composition stœchiométrique : on est ainsi conduit à examiner le diagramme thermique $\mathrm{Fe}_{3} \mathrm{O}_{4}-\mathrm{Fe}_{2} \mathrm{O}_{3}$. D'après les travaux de Huggett et Chaudron ( $\left.{ }^{4}\right)$,

(1) Cf. $\S$ i 2 et suivants.

(2) A paraître en 1949 dans les Annales de Géophysique.

(3) Phys. Rev., 1916, 8, 721 .

(*) C. R. Ac. Sc., 1927, 184, 201. 
Pfeil ( $\left.{ }^{1}\right)$, Meunier et Bihet $\left({ }^{2}\right), \mathrm{Fe}_{2} \mathrm{O}_{3}$ dissout à haute température jusqu’à 6 o/o de $\mathrm{Fe}_{3} \mathrm{O}_{4}$. mais ce te limile de solubilité diminue lorsque la température s'abaisse. Le refroidissement de la solution solide est danc accompagné d'une précipitation de magnélile.

On est donc amené à se demander si les axes de cristallites formant le précipité ne seraient pas orientés d'une manière bien déterminée par rapport aux axes de la matrice de $\mathrm{Fe}_{2} \mathrm{O}_{3}$.

L'épitaxie spinelle-oligiste. - Les minéralogistes ont observé depuis longtemps l'existence d'accolements réguliers entre cristaux d'espèce différente, naturels ou artificiels. qui sont ainsi mutuellement orientés : c'est le phénomène de l'épılaxie $\left({ }^{3}\right)$. Précisément dans le cas qui nous occupe, Royer $\left(^{4}\right)$ signale que plusieurs auteurs ant observé, notamment parmi des produits d'éruption volcanique; " des groupements réguliers de spinelle et d'oligiste qui se font de " manière qu'une face (ooor) de l'oligiste soit parallèle à une " face (lll $\left.\begin{array}{ll}1 & 1\end{array}\right)$ du spinelle »; ce spinelle peut être de la magnétite plus ou moins pure. Ces accolements ont probablement pris naissance au cours d'un refroidissement extrêmement lent d'une solution solide $\mathrm{Fe}_{2} \mathrm{O}_{3}-\mathrm{Fe}_{3} \mathrm{O}_{4}$.

Les structures de Widmanställen. - Au surplus, l'étude micrograpbique des alliages fournit de nombreux exemples d'orientations du même genre : il s'agit des structures de Widmanstätlen $\left(^{5}\right)$. Non seulement la direction des axes des cristallites mais aussi leur forme extérieure sont en rapport étroit avec les axes de la matrice; par exemple, les cristallites affectent souvent la forme de plaquettes minces, parallèles à des plans cristallographiques bien déterminés de la matrice.

7. Existence probable d'une phase de transition. - Il semble tout à fait vraisemblable, d'après les considérations précédentes, d'admettre que les cristallites de magnétite, qui se forment au cours du refroidissement du cristal d'hématite, présentent un axe ternaire parallèle à l'axe ternaire de la matrice.

Cependant cette hypothèse ne résout pas du tout les difticultés car, d'une part l'axe ternaire de la magnétite est une direction de facile aimantation, tandis que d'autre part l'anisotropie magnétocristal-

(1) J. Iron Steel Inst., r93 г 123, $237 ;$ г $929,119,501$.

( $\left.{ }^{2}\right) 15 \mathrm{e}$ Congrès Chimie Industr., 1935 .

( $\left.{ }^{3}\right)$ Consulter à ce sujet la belle thèse de Louis Royer (Strasbourg, 1928): Recherches expérimentales sur l'épilaxie. Elle m'a été sigoalée par M. J. Wyart, Professeur à la Sorbonne, que je tiens à remercier ici.

(4) Loc. cit, 134

(5) Voir par exemple: C. S. Barnetr. Structure of Metals (Mc Graw Hill Book, New.York, I943), ch. XXII. 
line de la magnétite, cristal cubique, est considérablement plus faible que les fortes anisotropies mises en évidence dans les expériences de Townsend Smith. On pourrait aussi songer à l'anisotropie de forme que présenteraient des cristallites ayant la forme de plaquetles minces perpendiculaires à l'axe ternaire, mais, là aussi, l'effet est trop faible pour interpréter les résultats expérimentaux. De plus, le point de disparition des propriétés ferromagnétiques de l'hématite en question est à $675^{\circ} \mathrm{C}:$ Il diffère donc considérablement du point de Curie de la magnétite, situé à $590^{\circ} \mathrm{C}$. L'impureté ferromagnétique n'est donc pas de la magnétile ordinaire.

On peut alors se demander si la formation finale du précipité de magnétite ne serait pas précédée de la formation d'une phase de transitıon dont les propriétés seraient nettement différentes.

Les phases de iransition dans les alliages. - De telles phases sont bien connues : elles provoquent le durcissement structural. En effet, l'expérience montre que lorsqu'il existe des relations entre les orientatious cristallines du précipité et de la matrice, les plans atomiques en regard, de part et d'autre de la surface qui sépare la matrice du précipité, possèdent des structures voisines aussi bien en ce qui concerne la disposition géométrique des atomes qu'en ce qui concerne leurs distances mutuelles. Il est donc tentant de supposer (1)qu'après une diffusion initiale des atomes, le précipité prend naissance de manière à adapter son réseau cristallin à celui de la matrice, notamment en ce qui concerne les distances mutuelles des atomes, comptées parallèlement au plan d'accolement. Naturellement, cette adaptation des deux réseaux entraine l'apparition de tensions internes considérables. Comme l'ont montré Mott et Nabarro $\left({ }^{2}\right)$, le précipité doit se développer en plaquette mince, forme qui réduit au minimum l'énergie de déformation élastique. Dès que l'épaisseur de cette plaquette dépasse une certaine valeur critique, les tensions internes deviennent suffisantes pour rompre la continuité entre les deux réseaux accolés; les tensions internes se relâchent et le précipité prend son réseau final normal.

Des phénomènes de ce genre ont été mis en évidence pour la première fois sur le système $\mathrm{Al}$ - Cu dans des expériences mémorables dues à Guinier $\left({ }^{3}\right)$. De nouveaux exemples ont été étudiés depuis,

(1) Cf. Barrett. Loc. cit.

( $\left.{ }^{2}\right)$ N. F. Mott et F. R. Nabarro. Proc. Phys. Soc. (London), 1940, 52, 86; F. R. Nabarro Proc. Phys. Soc. (London), 1940, 52, 90 ; Proc. Roy. Soc. (London), 1940, AI 75, 519; Symposium on Internal Stresses in Melals and Alloys, London, 1947,237 .

(3) A. Guinier. C. R. Ac. Sc., 1937, 204, 1115 ; J. de Phys. Rad., 1942, 3, 124; G. D. Preston. Nalure. 1938, 142, 570; Phil. Mag., 1938, 26, 855; Proc. Phys. Soc. London, i940, 52, 77; Proc. Roy. Soc. London, 1938, A 167, 526; Gayler. J. Inst. Melals, $1946,72,243$; Mehl et Jetrar. Amer. Soc. Metals, "Age-Hardening of Metals n, Cleveland, O., 1940. 
comme celui du système $\mathrm{Al}-\mathrm{Ag}\left({ }^{1}\right)$. Dans cet exemple, le précipité constitue une phase $\gamma$ hexagonale compacte (fig. I G) accolée, suivant le plan de base (ooor), parallèlement à un plan (i I 1 ) de la matrice qui est cubique à faces centrées (fig. I A), tandis que dans le plan d'accolement les rangées d'atomes les plus denses de la matrice et du précipité sont parallèles. De part et d'autre du plan d'accolement, et parallèlement à lui, les atomes forment un arrangement hexagonal plan, avec une distance entre atomes voisins de $2,858 \AA$ pour la matrice et de 2,879 A pour le précipité. Mais, cette dernière distance correspond à la phase finale du précipité : l'expérience a montré en effet l'existence d'une phase intermédiaire $\gamma^{\prime}$ qui est également hexagonale compacte (fig. I B) mais dans laquelle les distances inter-

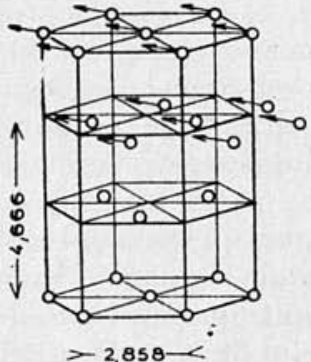

A

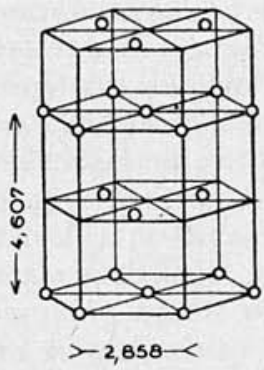

B

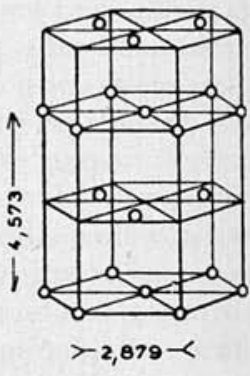

C

Fig. 1. - Mécanisme de la précipitation dans le système $\mathrm{Al}-\mathrm{Ag}$.

En $A$, réseau cubique de la matrice ; en $\mathrm{C}$, phase $\gamma$ finale du précipité, hexagonale compacte; en B, phase $\gamma^{\prime}$ intermédiaire, hexagonale compacte, adaptée sur le réseau de la matrice (distances en angströms).

atomiques dans le plan de base sont de $2,858 \AA$, c'est-à-dire telles que le réseau cristallin du précipité s'ajuste exactement sur celui de la matrice. Le précipité conserve la forme $\gamma^{\prime}$ tant que l'épaisseur des plaquettes du précipité n'est pas trop grande. Au delà d'une certaine épaisseur, qui est de quelques centaines d'angströms, la rupture se produit sous l'influence des tensions internes: le réseau du précipité se disjoint de celui de la matrice et prend la forme $\gamma$ en même temps que les tensions internes se relâchent.

Un mécanisme du même genre doit être à l'origine de la formation des groupements épitaxiques, car leurs conditions d'apparition sont analogues à celles des structures de Widmanstätten : en effet, Royer $\left({ }^{2}\right)$ a montré que, dans le plan d'accolement, les mailles planes

(1) A. Guinier. Loc. cit.; Geisler, Barrett et Merl. Trans. Amer. Inst. Min. Met. Eng., 1943, 152, $182 ; 1941,143,134$.

${ }^{(2)}$ Loc. cit., $\$ 6$. 
des deux espèces doivent être quasi identiques en ce qui concerne la disposition géométrique des atomes, leurs distances mutuelles et leur polarité.

Examinons de ce point de vue les structures cristallines de la magnétite et de l'hématite.

8. Les structures cristallines $\left({ }^{1}\right)$. - Structure de $\mathrm{Fe}_{3} \mathrm{O}_{4}$. - Elle peut être considérée comme formée de couches d'atomes d'oxygène et de couches d'atomes de fer, empilées alternativement perpeudiculairement à l'axe ternaire [ $\left.\begin{array}{lll}1 & 1\end{array}\right]$. La distance entre deux couches de même espèce est de 2,42 A (fig. 2). Les couches $a$ d'oxygène sont planes avec arrangement hexagonal compact des atomes et distance entre proches voisins égale à 2,97 ̊. Quant aux couches d'atomes de fer, elles appartiennent à deux catégories $b$ ou $b^{\prime}$. Les couches $b$ sont planes et les atomes placés comme l'indique la figure 3 : c'est un arrangement hexagonal compact dans lequel il manque un atome sur quatre. Les couches $b^{\prime}$ contiennent le même nombre d'atomes de fer que les couches $b$, mais les $2 / 3$ des atomes sont déplacés par rapport aux positions $b$, à la fois dans le plan et dans une direction perpendiculaire : la couche n'est plus plane.

Structure de $\mathrm{Fe}_{2} \mathrm{O}_{3} \alpha$. - Elle e-t encore constituée de couches d'atomes d'oxygène et de couches d'atomes de fer, empilées alternativement (fig. 4) parallèlement au plan de base (0001): la distance entre deux couches d'atomes semblables est de 2,29 $\AA$. Les couches $a^{\prime}$ d'atomes d'oxygène sont planes et dérivent d'un assemblage compact, analogue à celui de la couche $a$ de $\mathrm{Fe}_{3} \mathrm{O}_{4}$, au moyen de

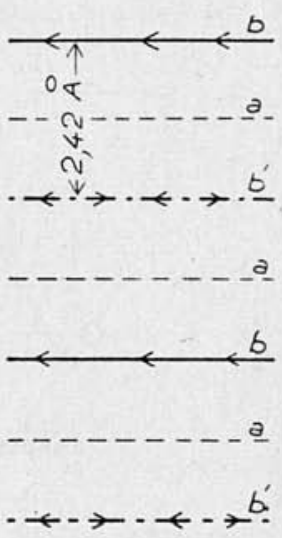

Fig. 2. - Magnétite.

Couches successives perpendiculaires à l'axe ternaire d'atomes de fer $\left(b, b^{\prime}\right)$ et d'atomes d'oxygène $(a, a)$. Les flèches correspondent aux directions d'aimantation spontanée. petits déplacements, de l'ordre de o, $3 \AA$, donnés dans des sens convenables aux différents atomes : la distance interatomique moyenne dans ce plan est de 2,90 A. Enfin, dans les couches $c$, les atomes de fer ont la disposition de la figure 5 , avec une distance interatomique moyenne de 2,9o $\AA$, mesurée en projection sur le plan moyen de la couche : en effet, celle-ci n'est pas plane, les atomes de fer étant situés alternativement au-dessus et au-dessous du plan moyen, à une distance de $0,29 \AA$.

(1) Cf. Ewald. Struklur Bericht. 
Remarque. - Les différentes couches $b, b, b, \ldots ; b^{\prime}, b^{\prime}, b^{\prime}, \ldots$, etc..., ne sont identiques qu'à des translations près dans leur plan qui ne nous intéressent pas ici.

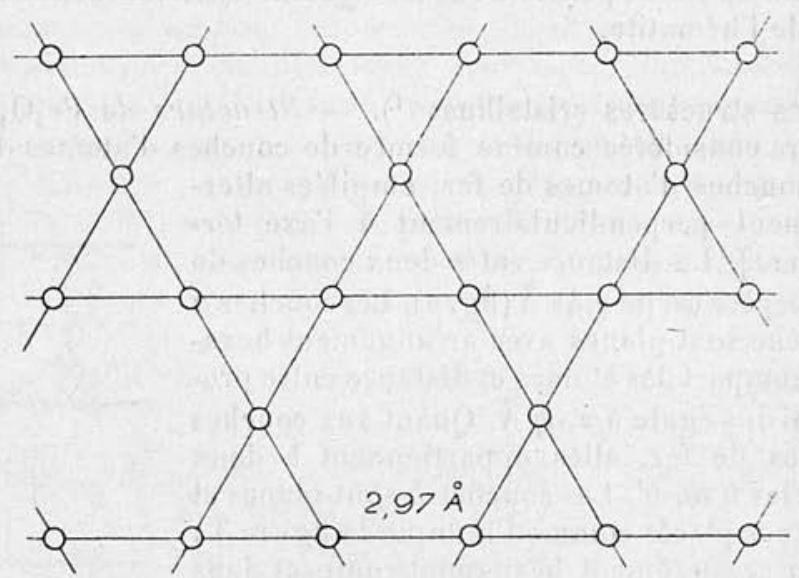

Fig. 3. - Magnélite.

Disposition des atomes dans les couches $b$ de fer.

Cette brève étude faït ressortir l'analogie de structure entre les couches de fer et surtout entre les couchesd'oxygène des deux oxydes: les atomes y sont placés de la même façon les uns par rapport aux

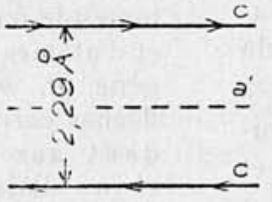

$-------a^{\prime}$

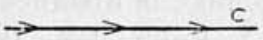

$------a^{\prime}$

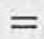

Fig. 4. - Hématite.

Couches successives perpendiculaires à l'axe ternaire d'atomes de fer $\left(c, c^{\prime}\right)$ et d'atomes d'oxygène $\left(a, a^{\prime}\right)$. Les flèches correspondent aux directions d'aimantation spontanée.

autres et leurs distances mutuelles moyennes, projetées sur le plan moyen, diffèrent relativement peu : 2,97 A dans le cas de la magnétite et $2,90 \AA$ pour $\mathrm{Fe}_{2} \mathrm{O}_{3} \alpha$. Les conditions nécessaires à l'épitaxie sont bien réunies. 
9. Mécanisme de la précipitation de magnétite. - On peut alors suggérer le mécanisme suivant pour la précipitation de la magnétite, à partir d'une solution solide à base de $\mathrm{Fe}_{2} \mathrm{O}_{3}$ sursaturée en magnétite. La matrice possède, à haute température, le même réseau cristallin que $\mathrm{Fe}_{3} \mathrm{O}_{3} x$ pur, à cette différence près que quelques atomes de fer manquent çà et là. A la suite de la diffusion des atomes de fer, facilitée par la nature lacunaire de leur réseau, et de légers glissements des a'omes d'oxygène parallèlement au plan de base, un précipité de $\mathrm{Fe}_{3} \mathrm{O}_{4}$ apparait sous forme de plaquettes minces parallèles au plan de base (ooor). Mais la continuité initiale nécessaire entre le

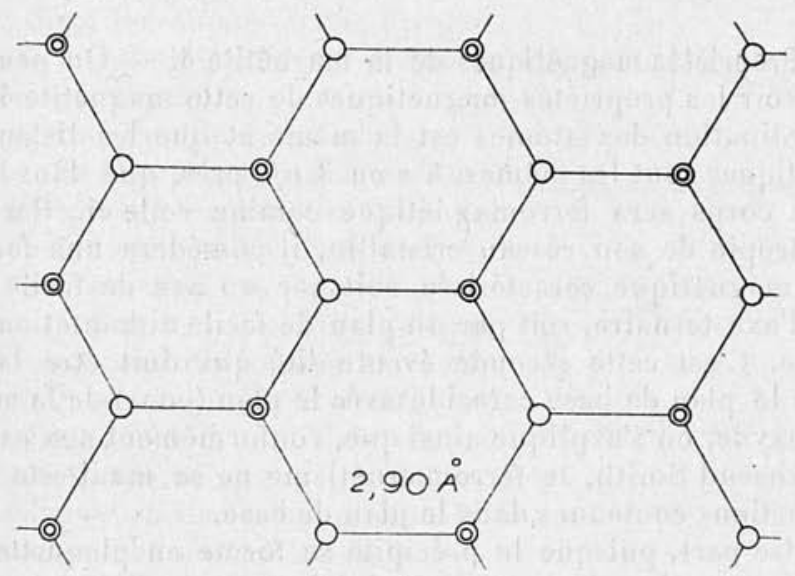

Fig. 5. - Hématile.

Disposition dis atomes dans les couches $c$ de fer; les cercles simples correspondent aux atomes situés à $0,29 \AA$ au-dessus du plan de figure; les doubles cercles aux atomes situés à $0,29 \AA$ au-dessous.

réseau de ce précipité et celui de la matrice ne permet pas au précipité de prendre le réseau cubique de la magnétite : le précipité prend un réseau rhomboédrique, dérivé de celui de la magnétite par une contraction dans le plan (lili) d'accolement, de manière à ramener la distance des atomes d'oxygène, qui est normalement de 2,97 $\AA$ dans la magnétite, à 2,9o $\AA$ distance moyenne des atomes d'oxygène dans le plan de base de $\mathrm{Fe}_{2} \mathrm{O}_{3} x$. Corrélativement, une dilatation se produit dans la direction de l'axe ternaire.

La magnétite $\delta$. - Nous appellerons magnétite $\delta$ cette phase intermédiaire rhomboédrique. Lorsque l'épaisseur des plaquetles de cette phase dépasse une certaine limite, il y a rupture de la continuité entre les deux réseaux et relâchement des tensions : le précipité se trans- 
forme en magnétite cubique ordinaire. Pendant cette transformation, les plans atomiques restent parallèles à eux-mêmes et on obtient une association des deux réseaux identique à celle des groupements épitaxiques naturels de magnétite et d'oligiste.

Nous súpposerons que le sesquioxyde de fer ordinaire est en réalité à haute température une solution solide $\mathrm{Fe}_{2} \mathrm{O}_{3}-\mathrm{F}_{3} \mathrm{O}_{4}$ dans laquelle la proportion de magnétite est très faible, de l'ordre de $10 / 0$, si bien que dans la précipitation qui se produit à basse température, l'épaisseur des plaquettes de magnétite reste toujours trop faible pour que la transformation en forme cubique se produise : le précipité conserve toujours la forme intermédiaire rhomboédrique.

10 Propriétés magnétiques de la magnétite $\delta$. - On peut essayer de prévoir les propriétés magnétiques de cette magnétite $\delta$ : comme la coordination des atomes est la même et que les distances ferromagnétiques sont les mêmes, à 2 ou 3 o/o près, que dans la magné. tite, ce corps sera ferromagnétique comme relle.ci. Par suite de l'anisotropie de son réseau cristallin, il possédera une forte anisotropie magnétique caractérisée. soit par un axe de facile aimanta. tion : l'axe ternaire, soit par un plan de facile aimantation : le plan de base. C'est cette seconde éventualité qui doit être la bonne: comme le plan de base coïncide avec le plan (ooor) de la matrice de sesquioxyde, on s'explique ainsi que, conformément aux expériences de Townsend Smith, le ferromagnétisme ne se maniteste que dans les directions contenues dans le plan de base.

D'autre part, puisque le précipité se forme en plaquettes minces parallèles au plan de base, la pulvérisation de $\mathrm{Fe}_{2} \mathrm{O}_{3}$ en poudrede plus en plus fine n'affecte vraisemblablement pas l'épaisseur des plaquettes mais seulement leurs dimensions latérales qui deviennent de plus en plus petites : on s'explique ainsi que Chevallier (') ail observé que le champ coercitif des poudres d'hématite ne dépendait que de la largeur des lamelles d'hématite et non pas de leur épais. seur.

11. Le point de Curie ferromagnétique de la magnétite $\delta$. - Tout d'abord, il ne faut pas s'étonner que le point de Curie de la magné. tite $\delta$ diflère de celui de la magnétite ordinaire puisque les distances interatomiques sont différentes. Il est plus remarquable de constater qu'il coïncide à $67^{\circ} \mathrm{C}$ avec le point de transition antiferromagnétique de $\mathrm{Fe}_{2} \mathrm{O}_{3} \alpha$. Il s'agit peut-être d'un hasard mais les interactions magnétiques entre la matrice et le précipité peuvent n'y être pas étrangères.

(') R. Chevallier et S. Mathieu. Ann. de Phys., 1943, 18, 258 (voir sur tout p. 270). 
Par suite de la continuité entre les réseaux de la matrice et du précipité, il existe des couplages de Weiss-Heisenberg entre les moments magnétiques des atomes de fer qui appartiennent aux deux couches de fer situées de part et d'autre du plan d'oxygène qui joue lêôle de paroi de séparation entre les deux phases. Ce sont : soit des actions directes entre atomes de fer seion le mécanisme d'échange de Heisenberg, soit des actions indirectes selon le mécanisme de superchange proposé par Kramers ( $\left.{ }^{1}\right)$. D'autre pait, d'après des travaux récents de L. Néel $\left({ }^{2}\right)$, l'antiferromagnétisme du sesquioxyde de fer rhomboédrique proviendrait de la déformation sous l'action du champ magnétique d'un assemblage à moment résultant nul composé de couches de fer successives, parallèles au plan de base, aimantées spontanément, alternatirement dans ún sens et dans. le sens inverse. En supposant par exemple négatives lesinteractions entre les deux réseaux, on peut schématiser par la figure 6 l'aimantation des couches successives de fer du système matrice-précipité.

En somme, la direction de l'aimantation spontanée de la magnétite $\delta$ est couplée à la direction d'antiferromagnétisme de la matrice. Or les moments magnétiques des atomes de la matrice conservent une orien'tation ordonnée jusqu'à $67^{\circ} \mathrm{C}$, point de disparition de l'ordre antiferromagnétique. Par suite des interactions entre les deux réseaux, l'ordre qui règne dans la matrice contribue à maintenir l'ord re dans le précipité, c'est-à-dire le parallélisme mutuel des moments élémentaires : ce sont ces actions extérieures qui élèvent le point de Curie de la magnétite $\delta$ pour l'amener à $67^{\circ} \mathrm{C}$.

Une autre façon? de présenter les choses consiste à considérer le șstème matrice-précipité comme un antiferromagnétique imparfait dans lequel les deux phases aimantées en sens iuverse ne posséde-

(1) H. A. Kramers. Physica, ${ }_{9} 34,1,182$; Réunion d'études sur le Magnétisme, Strasbourg, $19^{39}, 3,45$.

(7) L. NÉEl. A nn. de Phys., 1948, 3, 137 . 
raient pas des moments magnétiques tout à fait égaux. Nous avons montré (1) qu'il apparaît alors un ferromagnétisme d'un type spécial (ferrimagnétisme) dont le point de Curie ferromagnétique coïncide pratiquement avec le point de transition antiferromagnétique pur que présenterait la substance si les deux phases possédaient des moments égraux.

Les difficultés signalées sont donc levées.

12. Variation du champ coercitif en fonction des dimensions des grains. - Le champ coercitif de l'hématite dépend fortement de l'état physique et notamment de la grosseur du grain. R. Chevallier a obtenu $\left({ }^{2}\right)$ des champs coercitifs croissant d'abord régulièrementà mesure que les dimensions des grains diminuaient, depuis 200 à 400 oersteds pour des diamètres de 200 à $300 \mu$, pour atteindre 1500 oersteds pour des diamètres de i $5 \mu$. Pour des diamètres plus faibles, il a observé une diminution du champ coercitif accompagnée d'une diminution de l'aimantation maximum.

Ces deux diminutions ne parai-sent pas présenter une significa. tion fondamentale : elles peuvent provenir soit de l'insuftisance des champs magnétiques utilisés pour saturer, soit d'une altération chimique provoquée par le broyage. Il se pourrait en effet que ce broyage soit accompagné d'une oxydation et par conséquent, conformément aux résultats de Snoëk (cf. \$ 3 ), d'une diminution du ferromagnétisme. D'ailleurs, Mlle Roquet $\left({ }^{3}\right)$ a étudié un échantillon de $\mathrm{Fe}_{2} \mathrm{O}_{3} x$ préparé par voie humide et recuit seulement à $700^{\circ} \mathrm{C}$ : le diametre des grains devait être plutôt de l'ordre de I plutôt que de ro microns; le champ coercitif atteignait dans ces conditions 7600 œrsteds.

De telles variations du champ coercitif avec les dimensions des grains ont été très fréquemment observées : Gottschalk $\left(^{4}\right)$ sur la magnétite, Guillaud ( ${ }^{5}$ ) sur le manganèse-bismuth ont trouvé des champs coercitifs variant à peu près comme l'inverse du diamètre des grains et atteignant aussi des valeurs considérables : 12000 œrsteds pour des particules de $\mathrm{Mn}-\mathrm{Bi}$ de 3,3 microns. On sait d'autre part que le fer, en grains de quelques centaines d'angströms de diamètre, possède un champ coercitif atteignant $1000 œ r s t e d s\left({ }^{6}\right)$.

(1) L. NÉEl. Ann. de Phys., 19 $98,3,137$.

(2) Loc. cit., 278.

(3) C.R.Ac.Sc., $1947,224,1418$.

(4) V. Gotrschalk. Physics, vg35, 6, 127 ; V. Gotrschalk et F. Wart. Mans. $U$. S. Bu. Mines, Rep. Investig., no $3268,1935,67$ et 83.

(5) These Strasbourg, 1943.

(6) L. NÉEe, L. Weit, J. Aubry. Brev. Fr. dép. Chambéry, no pv. 323, ; avril 1942. 
Les valeurs considérables du champ coercitif des substances ferromagnétiques extrêmement divisées s'expliquent aisément ( ${ }^{1}$ ) par le fait que le processus ordinaire de désaimantation, par déplacement des parois de séparation entre les domaines élémentaires, ne peut évidemment se produire quand les dimensions des grains deviennent inférieures à l'épaisseur de paroi qui est de l'ordre du dixième de micron dans le fer. Le champ coercitif est alors déterminé, selon les cas, par l'anisotropie de forme $\left({ }^{2}\right)$ ou par l'anisotropie magnétocristalline $\left({ }^{3}\right)$. On sait donc à peu près ce qui se passe pour les grains très fins, malheureusement aucune théorie adéquate ne rend compte quantitativement des phénomènes dans le domaine de transition des grains de dimensions moyennes.

Quoi qu'il en soit, il parait légitime d'admettre que le mécanisme d'augmentation du champ coercitif de $\mathrm{Fe}_{2} \mathrm{O}_{3} \alpha$ avec la division des grains est le même que pour les autres ferromagnétiques et ne pose pas de problème spécial.

13. Aimantation à chaud du sesquioxyde de fer. - L'oxyde de fer $\mathrm{Fe}_{2} \mathrm{O}_{3} \alpha$ présente le phénomène de l'aimantation à chaud d'une

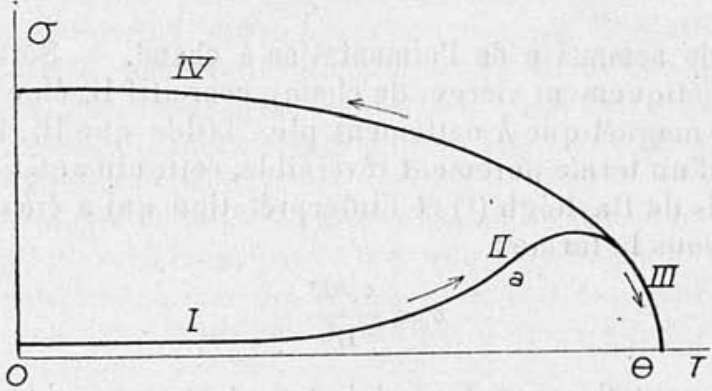

Fig. 7. - Courbes thermomagnétiques

du sesquioxyde de fer rhomboédrique (anomalie $a$ de Michel).

manière très accentuée $\left({ }^{4}\right)$. La courbe I (fig. 7) représente par exemple la courbe d'aimantation, en fonction de la température, dans un champ constant de l'ordre d'une centaine d'œrsteds, d'un échantillon de $\mathrm{Fe}_{2} \mathrm{O}_{3} x$ préparé par voie humide et recuit I heure à $1000^{\circ} \mathrm{C}$.

(') L. NÉEL. G.R. Ac. Sc., 1947, 224, 1488.

(') L. NÉEL. C. R. Ac. Sc., 1947, 224, 1550; L. WeIL. C. R. Ac. Sc., $1947,225,229$.

(3) L. NÉEL. G. R. Ac. Sc., 1947, 224, 1488.

(4) Cf. la thèse de A. Michel. Ann. de Chim., $1937,8,317$ où l'on trouvera les renseignements les plus complets sur Yaimantation 'à chaud, ainsi qu'une bibliographie. 
A température croissante l'aimantation, d'abord constante, croît nettement à partir d'une certaine température (anomalie $a$ ), atteint un maximum puis tend vers zéro au point de Curie. Au retour, l'aimantation augmente d'une manière continuelle à mesure que la température s'abaisse (courbe IV) et atteint à la température ordinaire une valeur beaucoup plus grande que la valeur initiale. Les chauffages ultérieurs donnent des courbes qui se confondent avec la courbe IV, tant à l'aller qu'au retour.

A. Michel (1) rattache ces phénomènes à la grande valeur de la dureté magnélique de la substance, cette dureté étant définie par le coefficient $a$ de la loi d'approche à la saturation :

$$
\sigma=\sigma_{\mathrm{s}}\left(\mathrm{I}-\frac{a}{\mathrm{H}}\right)
$$

et à l'existence d'un champ structural. Il nous semble plus simple de suivre l'idée de H. Forestier $\left({ }^{2}\right)$ et de rattacher directement l'ai. mantation à chaud à la grandeur du champ coercitif $\mathrm{H}$. On sait d'ailleurs que la dureté $a$ n'est pas forcément liée au champ coercitif : les fers frittés possèdent par exemple une grande dureté magnétique et un faible champ coercitif $\left({ }^{3}\right)$.

14. Théorie sommaire de l'aimantation à chaud. - Soit une sul. stance magnétiquement vierge, de champ coercitif $\mathrm{H}_{c}$ élevé, soumise à un champ magnétıque $h$ nettement plus faible que $\mathrm{H}_{c}$. En faisant abstraction d'un terme purement réversible, cette aimantation s'écrit, selon les lois de Rayleigh $\left({ }^{4}\right)$ et l'interprétation qui a été donnée de ces lois $\left({ }^{5}\right)$, sous la forme :

$$
\sigma=\frac{\sigma_{s} k h_{c}^{2}}{H_{c}^{2}}
$$

où $\sigma_{s}$ est l'aimantation spontanée à la tempéralure considérée et $k$ un coefficient numérique de l'ordre de quelques dixièmes, constant pour un échantillon donné. Lorsque la température s'élève, $\sigma_{\mathrm{s}}$ et $\mathrm{H}_{c}$ décroissent mais l'expérience montre que $\mathrm{H}_{c}$, et a forliori $\mathrm{H}_{c}^{2}$, décroissent beaucoup plus rapidement avec $\mathrm{T}$ que l'aimantation spontanée : il en résulte que l'aimantation $\sigma$, donnée par l'expression [3] où $h$ est constant, doit croître avec T. Cette variation, d'abord lente, s'accélère lorsque $\mathrm{H}_{c}$, à la suite de sa diminution ther-

(1) Loc. cit., 334 .

$\left({ }^{2}\right)$ C. R. Ac. Sc., 1935, 201, 45.

( ${ }^{3}$ L. NÉEl. C. R. Ac. Sc., 1945, 220, 738 ; J. de Phys. et Radium, 1948 , 9, 184 .

(4) Phil. Mag., $1887,23,225$.

(5) L. NéEl. Cahiers de Phys., 1942, no 12, 1; 1943, no 13, 18. 
mique, devient égal au champ appliqué $h$ : la loi [3] n'est plus valable et $k$ se rapproche de l'unité. Puis, lorsque $h$ devient plus grand que $\mathrm{H}_{c}, \sigma$ devient voisin de $\sigma_{s}$ et diminue avec la température comme cette quantité (cf. fig. 7 , courbe III). En somme l'anomalie $a$ correspond à la température à laquelle le champ coercitif devient égal au champ appliqué. On s'explique ainsi notamment que le recuit abaisse la température de l'anomalie $a($ (') puisque le recuit abaisse l'ensemble de la courbe $\mathrm{H}_{c}=f(\mathrm{~T})$.

Lorsqu'en effectuant des recuits prolongés $\left(6\right.$ heures à $\left.1300^{\circ} \mathrm{C}\right)$, on abaisse la valeur du champ coercitif à la température ordinaire au-dessous de la valeur du champ appliqué $h$, l'anomalie $a$ disparait: l'aimantation $\sigma$ reste toujours de l'ordre de grandeur de $\sigma_{\mathrm{s}}$ el varie avec la température comme $\sigma_{s}$.

De même, H. Forestier $\left({ }^{2}\right)$ a constaté que l'aimantabilité à chaud était liée à la finesse du grain : la dimension des grains de $\mathrm{Fe}_{2} \mathrm{O}_{3} \alpha$ devant ètre inférieure à $0,07 \mathrm{~mm}$. pour que le phénomène se produisit. En réalité, il s'agit là d'un effet de l'augmentation du champ coercitif avec la finesse du grain. Les gros grains doivent posséder un champ coercitif plus petit que le champ appliqué $h$ et l'anomalie $a$ ne doit pas apparaître. D'après les expériences de Chevallier $\left({ }^{3}\right)$, des grains de $0,07 \mathrm{~mm}$. possèdent un champ coercitif de 500 œrsteds, ce qui est bien de l'ordre de grandeur des champs qui règnent dans l'entrefer des appareils thermomagnétiques employés par les auteurs cités dans l'étude de $\mathrm{Fe}_{2} \mathrm{O}_{3} \alpha$.

Enfin, la forme de la courbe d'aimantation, au cours du refroidissement, s'explique aisément par le fait que l'aimantation spontanée des domaines élémentaires de Weiss, une fois retournée dans la direction du champ, conserve au cours du refroidissement cette nouvelle orientation.

15. Généralité de l'aimantation à chaud. - La théorie que nous venons de développer montre que l'aimantation à chaud devrait constituer une propriété commune à tous les ferromagnétiques, à condition de les étudier dans un champ magnétique approprié. Un appareil thermomagnétique où règne un champ d'une centaine d'ersteds ne peut indiquer d'aimantation à chaud que pour des substances dont le champ coercitif est de plusieurs centaines d'œrsteds. En outre, pour les substances à faible champ coercitif et par conséquent à grande perméabilité, étudiées sous forme de poudres, la présence du champ démagnétisant interne de forme gêne l'acquisition de l'aimantation à chaud. Pour ces deux raisons, l'aimantation

(1) A. Michвl. Loc. cit., 323 .

(2) C. R. Ac. Sc., $1935,201,45$.

(i) Ann. de Phys., 1943, 18, $27^{8 .}$

Ann. de Phys., 12e Série, t. 4 (Mai-Juin 1949). 
à chaud n'attirera l'attention que pour les substances à grand champ coercitif, mais bien peu d'entre elles sont susceptibles d'être étudiées de ce point de vue. En effet la plupart des substances à grand champ coercitif sont des alliages à durcissement structural dont la structure se moditie complètement par chauffage à la température du point de Curie. Il en est de même pour les poudres fines métalliques à grand champ coercitif qui se frittent en général au-dessous du point de Curie. Finalement le phénomène ne doit guère être observable que sur les substances non métalliques à grand champ coercitif, comme les ferrites et le sesquioxyde de fer, pour lesquels, effectivement, l'aimantation à chaud a été observée.

16. L'anomalie A de Michel. - Certains échantillons de $\mathrm{Fe}_{2} \mathrm{O}_{12}$ recuits seulement à $700^{\circ} \mathrm{C}$ présentent ce que Michel a appelé l'ano.

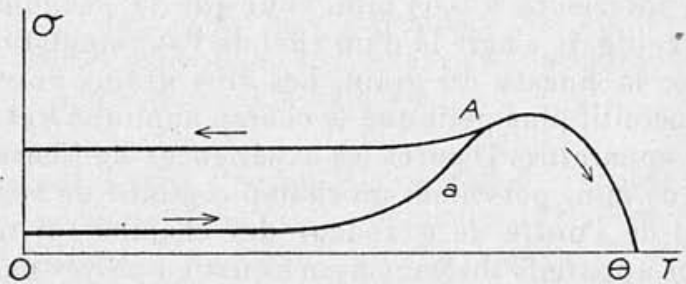

Fig. 8. - Courbes thermomagnétiques du sesquioxyde de fer rhomboédrique (anomalie A de Michel).

malie A $\left({ }^{1}\right)$ : elle consiste dans une diminution de l'aimantation, sur la courbe de refroidissement (cf. fig. 8).

Dans ces échantillons, le champ coercitif élevé provient probable. ment de l'effet de division $\left({ }^{2}\right)$ et doit être proportionnel à la constante d'anisotropie K. Cette constante K, dans les quelques exemples ćttdiés $(\mathrm{Fe}, \mathrm{Ni})$ varie très rapidement et devient très petite à $100^{\circ}$ ou $200^{\circ}$ du point de Curie $\left({ }^{3}\right)$ : une théorie d'Akulov $\left({ }^{4}\right)$ montre d'ailleurs qu elle varie comme $\sigma_{\mathrm{s}}^{10}$. Considérons alors un grain $\mathrm{G}$ et désignons par $\Delta$ la direction de facile aimantation la plus rapprochée du champ appliqué $h$. Du point de Curie jusqu'à $200^{\circ}$ au-dessous par exemple, la constante K reste très petite de sorte que $h \sigma_{\mathrm{s}}$ est grand devant K: l'aimantation du grain reste dirigée dans la direction du champ appliqué $h$. Au contraire, au-dessous de cet intervalle, les forces

(1) A. Michel. Loc. cil., $32 \mathrm{I}$.

(2) L. NÉEL. C. R. Ac. Sc., $1947,224,1488$.

( $\left.{ }^{3}\right)$ Cf. par ex. Beckrr. Ferromagnetismus, 125.

(4) N. Akulov. $Z . f$. Physik, 1929, 52, 389. 
magnétocristallines l'emportent devant les forces directrices dues au champ $h$ et l'aimantation spontanée regagne la direction $\Delta$ de facile aimantation la plus voisine, de sorte que la composante de l'aimantation suivant la direction du champ appliqué diminue. Comme le champ coercilif $\mathrm{H}_{c}$ est précisément de l'ordre de $\mathrm{K} / \sigma_{\mathrm{s}}\left({ }^{1}\right)$, il en résulte que la température de l'anomalie $\mathrm{A}$ doit être voisine de la température de l'anomalie $a$, conformément à l'expérience.

17. Aimantation à chaud des monocristaux d'oligiste. - H. Forestier $\left({ }^{2}\right)$ a constaté qu'un monocristal d'oligiste présentait une aimantation à chaud prononcée suivant l'axe ternaire tandis qu'il n'en présentait pas dans les directions perpendiculaires. Il faut évidemment rapprocher ce fait de ce que l'axe ternaire est une direction de très difficile aimantation. En effet, A. Michel $\left({ }^{3}\right)$ a montré que l'axe binaire de la pyrrhotine, qui est une direction de très difficile aimantation, est aussi la direction suivant laquelle l'aimantation à chaud est la plus prononcée.

On peut donner de ce phénomène l'interprétation suivante: lorsqu'une substance présente une direction de très difficile aimantation, toutes les variations d'aimantation que nous puissions produire au moyen des champs magnètiques techniquement accessibles proviennent en dernière analyse de la rotation de l'aimantation spontanée dans le plan perpendiculaire à la direction de très difficile aimantation et sont provoquées par la composante dans ce plan du champ magnétique appliqué. La composante agissante du champ est donc très faible quand la direction d'observation, que nous supposons coïncider avec celle du champ, est presque parallèle à la direction de très difticile aimantation : il en résulte que le champ coercitif apparent tend vers l'infini à mesure que l'on se rapproche de cette direction.

Dans le cas d'une poudre d'oligiste, composée de grains pas trop fins orientés au hasard, le champ coercitif moyen est trop faible pour que l'aimantation à chaud soit observable, mais pour un cristal orienté et étudié dans une direction voisine de celle de l'axe ternaire, le champ coercitif apparent devient suffisamment grand pour que l'aimantation à chaud soit notable.

On peut objecter que, dans la direction exacte de l'axe ternaire, l'aimantation rémanente observable est théoriquement nulle puisque celte direction est perpendiculaire aux directions de facile aimantation suivant lesquelles l'aimantation spontanée doit toujours s'orienter en l'absence de champ extérieur. En fait, les accidents du réseau

(') NÉEL, loc. cil.

(') C. R. Ac. Sc., $1935,201,45$.

(3) Cf. sa thèse, loc. cit. 
cristallin, qui existent d'ailleurs nécessairement puisqu'il existe un champ coercitif, perturbent les directions de facile aimantation et rendent possible la présence d'une faible composante d'aimantation rémanente dans la direction de l'axe ternaire.

18. Conclusions. - Il conviendrait maintenant d'achever cette revue des propriétés magnétiques du sesquioxyde de fer rhomboédrique par l'examen de ses proprictés dans les champs magnétiques très faibles, de l'ordre de grandeur du champ terrestre. E. Thellier et sa collaboratrice, Mlle J. Roquet, ont en effet mis en évidence dans ces conditions des propriétés très curieuses, soit dans l'étude du sesquioxyde de fer artificiel, soit dans l'étude de terres cuites dans des conditions telles que leur constituant magnétique essentiel fut $\mathrm{Fe}_{2} \mathrm{O}_{3} x$. Cependant ces propriétés appartiennent aussi aux basaltes et aux terres cuites en atmosphère réductrice dont la magnétite est le constituant magnétique principal : elles ne sont donc pas spécifiques du corps que nous avons étudié et sont plutôt liées à un état physique particulier d'extrême division. Comme leur interprétation exige d'autre part des développements théoriques assez étendus, nous avons préféré consacrer à leur étude un mémoire spécial qui paraitra incessamment dans une autre publication ${ }^{(1)}$.

(1) En ז949, dans les Annales de Géophysique. 